\title{
Plasticity and Fracture in Drying Colloidal Films
}

\author{
Lucas Goehring, ${ }^{1, *}$ William J. Clegg, ${ }^{2, \dagger}$ and Alexander F. Routh ${ }^{3,4, \dagger}$ \\ ${ }^{1}$ Max Planck Institute for Dynamics and Self-Organization, Am Fassberg 17, D-37077 Göttingen, Germany \\ ${ }^{2}$ Department of Materials Science and Metallurgy, University of Cambridge, Pembroke Street, Cambridge CB2 3QZ, United Kingdom \\ ${ }^{3}$ Department of Chemical Engineering and Biotechnology, University of Cambridge, Pembroke Street, \\ Cambridge CB2 3RA, United Kingdom \\ ${ }^{4}$ BP Institute for Multiphase Flow, University of Cambridge, Madingley Rise, Madingley Road, Cambridge CB3 OEZ, United Kingdom
} (Received 8 August 2012; published 7 January 2013)

\begin{abstract}
Cracks in drying colloidal dispersions are typically modeled by elastic fracture mechanics, which assumes that all strains are linear, elastic, and reversible. We tested this assumption in films of a hard latex, by intermittently blocking evaporation over a drying film, thereby relieving the film stress. Here we show that although the deformation around a crack tip has some features of brittle fracture, only $20 \%-30 \%$ of the crack opening is relieved when it is unloaded. Atomic force micrographs of crack tips also show evidence of plastic deformation, such as microcracks and particle rearrangement. Finally, we present a simple scaling argument showing that the yield stress of a drying colloidal film is generally comparable to its maximum capillary pressure, and thus that the plastic strain around a crack will normally be significant. This also suggests that a film's fracture toughness may be increased by decreasing the interparticle adhesion.
\end{abstract}

DOI: 10.1103/PhysRevLett.110.024301

PACS numbers: 46.50.+a, 62.20.fq, 82.70.Dd

Colloidal dispersions, such as those found in many paints or coatings, often crack as they dry. A wide range of crack patterns are observed, such as regular parallel cracks [1,2], wavy cracks [3], star cracks [4], spiral cracks [5,6], and interface cracks [7]. Typically, interpretation of these patterns is made by the well-established framework of elastic fracture mechanics (e.g., Refs. [8,9]). Several studies have attempted to invert the observed deformation fields around cracks to measure material properties such as toughness or elastic modulus, by reference to solutions of elastic theories, either in a linear $[4,7,10]$ or nonlinear [11] formulation. However, although one has recently succeeded in directly measuring the interfacial toughness of a film on a compliant substrate [7], we still lack any detailed measure of the fracture properties, or inherent toughness, of colloidal films.

Elastic fracture mechanics assumes reversibility, and predicts that a crack will completely close if it is returned to its unloaded state. Cracks in colloidal films, however, remain open after drying is complete, when the capillary forces that originally led to the fracture are absent [12]. Other features, such as a drying-rate dependence on the terminal speed of cracks in drying pastes, also appear to require a plastic response [13]. Despite this, there has been no rigorous investigation of the reversibility assumption that underlies much of our interpretation of cracks in drying colloidal films. Here we demonstrate, experimentally, the relative importance of elastic and plastic deformations during the cracking of a hard latex. The film behaves in some ways as a brittle material. However, plastic effects account for the majority of the deformation and energy release during fracture, similarly to the case of "brittle" polymer materials such as poly(methyl-methacrylate) [14]. We show how plasticity may be related to changes in the film's microstructure and give a simple scaling argument implying its ubiquity.

The geometry of a directionally drying film is shown in Fig. 1, with drying fronts parallel to the $y$ axis, advancing

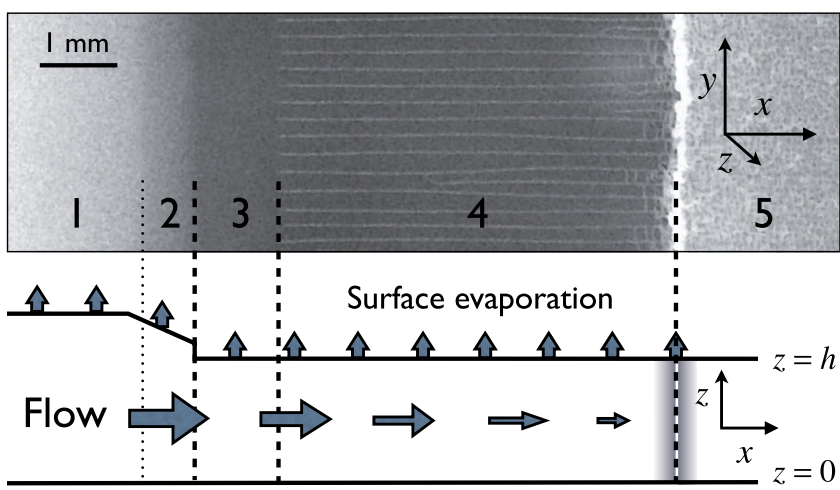

FIG. 1 (color online). Directional drying occurs when a thin colloidal film dries from its edges inward (e.g., Refs. [1,16,17,38]). A series of drying fronts form and travel toward (1) the stillliquid region of the film. As the material dries it first (2) orders, then (3) solidifies into an aggregated, porous particle network. Evaporation continues from the saturated film, balanced by the flow of dispersant toward the drying fronts from the liquid region. This flow is driven by a gradient in capillary pressure that also leads to (4) fracture. When the capillary pressure is large enough (5) the pores open and the film dries. Understanding the mechanical properties relevant to film fracture has proved challenging, as these properties are transitory, changing when the film dries into region (5). 
along the $x$ axis. The film's upper surface is exposed to air, and evaporating at a rate $\dot{E}$. Darcy's law, $\nabla p=-(\mu / \kappa) v$, describes how the resulting gradient of the capillary (or pervadic [15]) pressure drives flow of a fluid of viscosity $\mu$ at a superficial velocity $v$ in a film of permeability $\kappa$. Averaging over the height $h$ of the film, and considering flow only along the $x$ direction, mass conservation requires that $\partial v_{x} / \partial x=-\dot{E} / h$. Taking a derivative of Darcy's law then gives

$$
\frac{\partial^{2} p}{\partial x^{2}}=\frac{\mu \dot{E}}{\kappa h},
$$

which is frequently used to model pressure variations in drying films [16-19], and where the terms on the righthand side are usually taken to be constant across the saturated film (regions 3 and 4 in Fig. 1). Equation (1) predicts a spatially varying capillary pressure, whose magnitude at any particular point is proportional to the evaporation rate $\dot{E}[16-19]$.

Prior to cracking, the film is free to contract vertically in response to stress, but symmetry dictates that there is no displacement in the $y$ direction. With a no-slip boundary condition along the substrate and a traction-free upper film surface the equations of linear poroelasticity and stress equilibrium $[15,20,21]$ can be solved. When $d p / d x \ll$ $p / h$, the precrack total stress is an in-plane tensile stress proportional to the local pressure,

$$
\sigma_{x x}=\sigma_{y y}=-p \frac{1-2 \nu}{1-\nu},
$$

where $\nu$ is Poisson's ratio for the film.

A crack tip concentrates stress in its vicinity. The neartip variations in the total stress are characterised [8] by a stress intensity factor $K$. The critical stress intensity factor, or fracture toughness, $K_{C}$ represents a material's resistance to fracture: a crack will advance only if $K \geq K_{C}$, and a crack advancing quasistatically will balance $K=K_{C}$. In a drying colloidal film, crack tips advance rapidly to the line where the local stress is just sufficient to open them and then follow this line as the drying fronts advance. Under these conditions, for a through-thickness mode-I crack in a thin sheet, the opening displacement

$$
\delta=\frac{8 K_{C}}{E} \sqrt{r / 2 \pi}
$$

where $E$ is the Young's modulus of the film and $r$ is the distance along the crack from its tip [8,9]. Although Eq. (3) assumes the material is linear elastic, it also holds when irreversible deformation is confined to a small zone around the crack, for distances $r$ larger than the size of this zone [8]. Furthermore, for a channeling crack on a rigid substrate $\delta$ is expected to saturate when $r \gtrsim h[7,22,23]$. Thus, for the scaling of Eq. (3) to be observed, one must look at lengths larger than the plastic zone but smaller than the film thickness.
The crack opening $\delta$ was measured in drying colloidal films, by incident light microscopy with digital imaging. The films, typically a few tens of $\mu \mathrm{m}$ thick when dried, were spread onto the central region of $5 \times 7.5 \mathrm{~cm}^{2}$ glass slides and placed on the microscope stage. They dried naturally until a series of directional drying fronts formed, as in Fig. 1, including a fracture front with regularly spaced cracks. An individual crack was then selected, centered in the field of view, observed until it was seen to be intermittently advancing, and imaged. Images were thresholded to determine crack profiles, with the threshold value calibrated against manual measurements. Dry film thicknesses were measured by a scanning profilometer (Bruker DektakXT), and are accurate to $10 \%$. Dispersions of charge-stabilized colloidal polystyrene latex (described elsewhere [17,24]) were used, with particle radii $a$ of 49 , 58, 72, and $99 \mathrm{~nm}$ (Brookhaven ZetaPALS, dynamic light scattering, $5 \%$ accuracy), polydispersities up to $15 \%$, and solid volume fraction of $10 \pm 1 \%$.

As shown in Fig. 2, the opening displacements near crack tips agree with the parabolic shape predicted by Eq. (3). This agreement extends further in thicker films. The drying colloidal films thus have a well-defined fracture toughness. We calculated the ratio $K_{C} / E$ from Eq. (3), and a least-squares fit of a parabolic profile to $\delta(r)$. This material property has no strong particle size dependence (inset Fig. 2).

In order to evaluate the relative contribution of elastic and plastic effects to cracking, we investigated the reversibility of crack opening. After a crack tip had been imaged,

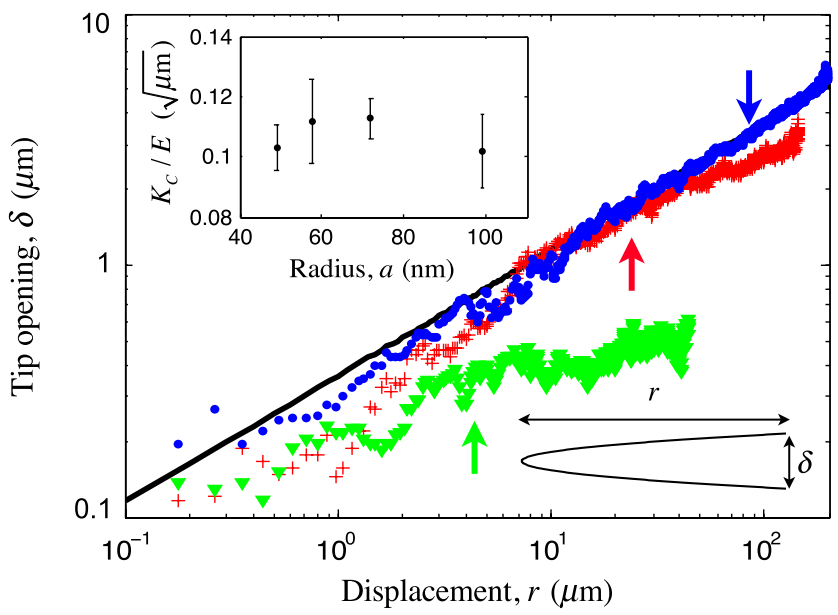

FIG. 2 (color online). The crack opening width $\delta$ varies as $\sqrt{r}$, near its tip. Shown are averages of at least ten crack widths with $a=72 \mathrm{~nm}$ in films with dried thicknesses of $5 \mu \mathrm{m}$ (green triangles), $26 \mu \mathrm{m}$ (red crosses), and $95 \mu \mathrm{m}$ (blue dots), and a best-fit square-root line to the thick-film data. Arrows indicate film thickness, after which deviations from this line are expected, as $\delta$ saturates. The inferred ratio (inset) of fracture toughness to Young's modulus, $K_{C} / E$, has no strong dependence on particle radius, over the range $50-100 \mathrm{~nm}$. 
a glass slide was slid into place $1 \mathrm{~mm}$ above, but not touching, the film, to block evaporation and relieve the in-plane stress described by Eqs. (1) and (2). For covered films, evaporation rates of order $10^{-9} \mathrm{~m} / \mathrm{s}$ are reported [25], an order of magnitude lower than over uncovered films [26]. A crack could be left covered for $15 \mathrm{~min}$ without noticeable growth, whereas for uncovered films a crack tip typically grew out of the microscope field of view within a minute.

When covered, the crack opening decreased, as demonstrated in Fig. 3(a). This closure occurred within the 5-10 s required to refocus on the crack tip, with no further opening or closing seen over 15 additional minutes. Observations were repeated several times on different films with particle radii 58, 72, and $99 \mathrm{~nm}$. There was considerable variation between individual cracks but no difference in reversibility between the different particle sizes. Cracks in films of each particle size were consistent with a $26 \pm 6 \%$ (mean \pm standard deviation) decrease in $\delta$ on covering. To confirm that this change was reversible, a crack was covered and uncovered multiple times. This crack repeatedly opened and closed, as shown in Fig. 3(b). The average closure, after the first cycle, was $20 \%$.

In further experiments, film stresses were reduced by adding water to the still-fluid central region of a drying film until it flooded over part of the saturated solid film, as in Fig. 3(c). This blocks evaporation over the watercovered section, but nothing is introduced that could change the image quality of the crack elsewhere. Here, $\delta$ was measured along the crack just prior to, and a few seconds after, flooding. The effects of flooding were statistically similar to covering; a $26 \pm 5 \%$ decrease in $\delta$ was observed. This degree of closure was observed both near
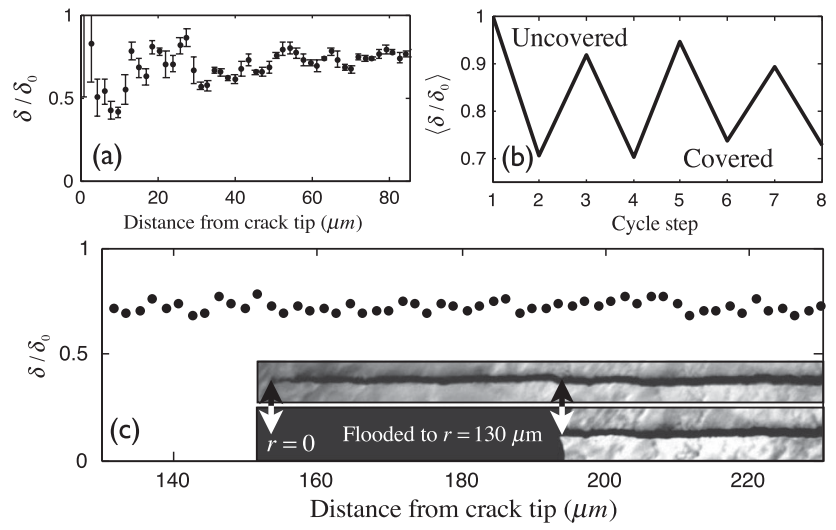

FIG. 3. Approximately $20 \%-30 \%$ of the crack opening $\delta$ is reversible. (a) When evaporation over a film of colloidal polystyrene $(a=58 \mathrm{~nm})$ is blocked by a glass slide, $\delta$ decreases from its stressed value $\delta_{0}$. (b) By repeatedly covering and uncovering the film, the crack repeatedly closes and opens. (c) A similar degree of closure can be achieved by flooding part of a film ( $a=72 \mathrm{~nm}$ ), eliminating evaporation locally. The inset shows the same crack before and after flooding, with the crack tip and extent of flooding indicated by arrows. crack tips themselves and hundreds of microns (many film thicknesses) away. Combining all results, we find that $70 \%-80 \%$ of the crack opening is irreversible and additional to the elastic response of the film to capillary pressure.

Irreversible, or plastic, deformation is frequently the result of changes to a material's microstructure [8]. To study this, drying colloidal films were imaged by atomic force microscopy (Veeco Dimension III, with RTESP tapping-mode cantilever). As the cracks advance episodically, rather than continuously [27], crack tips could remain stationary long enough to image their surroundings. Features such as microcracks [Figs. 4(a) and 4(b)], and bridging [Fig. 4(c)] were seen. These are classic crack-tip shielding features, known to enhance the toughness of materials such as ceramics and metals [8]. Finally, the region adjacent to a crack was observed, periodically, as the crack tip advanced. As demonstrated in Figs. 4(d) and 4(e), there were occasionally rearrangements of the particle network as drying proceeded. During these events particles changed neighbors, introducing irreversible strain. This mechanism was active away from the crack tip, but only in regions near crack faces. Control regions equidistant between two cracks showed no rearrangement during drying. This, and the near-tip crack opening data of Fig. 2, suggest that irreversible deformation is essentially confined to a plastic zone a few microns wide in the vicinity of the crack.

For a linear elastic material, the critical mechanical energy release rate $G_{C}$ of fracture is equal to the thermodynamically reversible work $W$ used to create the new crack surfaces. The addition of plastic losses modifies the energy balance, such that

$$
G_{C}=W+U_{p}
$$

where $U_{p}$ is the irreversible energy consumed per unit area of new crack interface [8]. This balance can also be expressed in terms of stress intensity factors. The correspondence between these views allows us to define both the actual fracture toughness $K_{C}=\sqrt{G_{C} E}$ as well as a fracture toughness pertaining to the reversible case, $K_{\text {rev }}=\sqrt{W E}$. Thus,

$$
\frac{K_{C}^{2}}{K_{\mathrm{rev}}^{2}}=1+\frac{U_{p}}{W}
$$

The reversible opening of a crack tip in a linear elastic solid should, in principle, follow

$$
\delta_{\mathrm{rev}}=K_{\mathrm{rev}} \frac{8}{E} \sqrt{r / 2 \pi}
$$

while the actual opening was shown, in Fig. 2, to follow the parabolic shape of Eq. (3). The ratio of the total crack opening $\delta$ to the reversible crack opening $\delta_{\text {rev }}$ is thus 

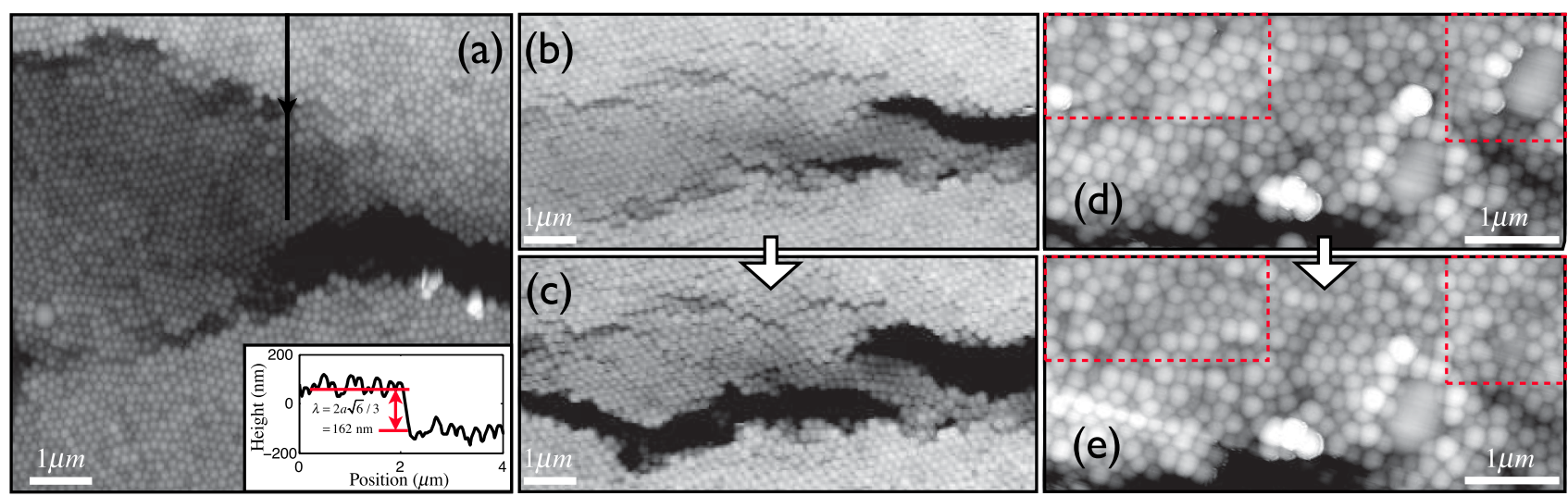

FIG. 4 (color online). Topographic atomic force micrographs of drying latex $(a=99 \mathrm{~nm}$ ). (a) A crack shows damage and microcracks ahead of the crack tip. Out-of-plane deformation is also noticed: the height profile across the overlain line segment (inset) shows a step in height of one stacking plane $(\lambda=2 a \sqrt{6} / 3)$. (b) Another crack shows a damage zone that develops into a bridge when (c) the crack advances. The particles (d) immediately adjacent to a crack face (the black region partly seen at the bottom of the image) rearrange when (e) the crack advances. Here the dashed boxes highlight two particular areas where a large particle submerges and where many particles rearrange.

$$
\frac{\delta}{\delta_{\mathrm{rev}}}=\sqrt{1+\frac{U_{p}}{W}} .
$$

We observed that only $20 \%-30 \%$ of the crack opening in our films was reversible, implying $U_{p} \gtrsim 10 \mathrm{~W}$. In other words, for a colloidal film of hard latex spheres the plastic work of fracture is significant.

The yielding behavior of aggregated colloidal dispersions is well studied (e.g., Refs. [28-32]). For an aggregated particle network, the yield stress $\sigma_{Y}$ is predicted to be

$$
\sigma_{Y}=\frac{\phi M F_{\max }}{4 \pi a^{2}},
$$

where $\phi$ is the solid volume fraction and $M$ the coordination number of the network, $a$ the radius of the particles, and $F_{\max }$ is the maximum interparticle force that can be sustained at any contact [28]. If this force is dominated by van der Waals adhesion between spherical particles, then for small separation $s$,

$$
F_{\max }=\frac{A a}{12 s^{2}}=2 \pi a \gamma_{p},
$$

where $A$ is the Hamaker constant and $\gamma_{p}$ the surface energy (half the work of adhesion) of the particle material [33]. For surfaces in intimate contact, $s$ is taken to be an atomic length scale [28]. This model has been successfully tested in a broad range of situations, including variations in particle size [31], volume fraction [28], chemical environment [29,30], and consolidation pressure [31].

To predict the scaling of the plastic contribution to fracture, we compare the yield stress to the maximum capillary pressure, $\sigma_{0} \sim \gamma / a$, generated by a fluid of surface tension $\gamma$ in a film containing particles of radius $a$ :

$$
\frac{\sigma_{0}}{\sigma_{Y}} \sim \frac{\gamma}{\phi M \gamma_{p}}
$$

If $\sigma_{0} / \sigma_{Y} \ll 1$, then plastic effects will be confined to a vanishingly small region around a crack tip, where stresses are concentrated enough to cause yielding. The energy $U_{p}$ consumed in this region will be small, and the crack will behave close to the brittle ideal. In the opposite limit, $\sigma_{0} / \sigma_{Y} \gg 1$, plastic yielding will no longer be confined to a small region around the crack tip, $U_{p}$ will be large, and the film will be tough. Polystyrene has a surface energy in air of $0.045 \mathrm{~J} / \mathrm{m}^{2}$ [34], but as its Hamaker constant is 6 times less in water [33] we take $\gamma_{p}=0.008 \mathrm{~J} / \mathrm{m}^{2}$. Using $\gamma=0.07 \mathrm{~J} / \mathrm{m}^{2}$ for water, and close-packed values of $\phi=$ $0.74, M=12$, for example, we estimate $\sigma_{0} / \sigma_{Y} \sim 1$ (or 2 for random-close packings of $\phi=0.64$ and $M=6$ ). Thus, the yield stress is comparable here to the applied stresses, for all particle sizes, arrangements, and drying conditions. Since this ratio is of order one it also suggests a simple way to control film fracture, by decreasing particle adhesion: here a small change to $\sigma_{0} / \sigma_{Y}$ should significantly change the plastic dissipation, increasing $G_{C}$. Indeed, a brittleto-plastic transition of this nature has been observed in colloidal alumina [30], when particle adhesion was varied by changing the surface chemistry. A similar transition would be predicted if the dispersant was replaced with one whose index of refraction more closely matched that of the particles, lowering $A$.

Finally, we consider the critical energy release rate $G_{C}$ of our films. While it remains a challenge to measure the elastic modulus of a colloidal film, during drying, a model has been developed $[35,36]$ for dry powder compacts,

$$
E=7.6 \phi^{4}\left(\frac{E_{0}^{2} \gamma_{p}}{a}\right)^{1 / 3}
$$


where $E_{0}$ is the Young's modulus of the particle material, $3 \mathrm{GPa}$ for polystyrene [37]. For $a=72 \mathrm{~nm}$, this predicts that $E$ is in the range $130-230 \mathrm{MPa}$, for packing fractions $\phi$ between 0.64 and 0.74 . As $G_{C}=\left(K_{C} / E\right)^{2} E$, and we have measured $K_{C} / E=0.11 \sqrt{\mu \mathrm{m}}$, the estimated energy release rate $G_{C}$ is $2 \pm 1 \mathrm{~J} / \mathrm{m}^{2}$, similar to that of silicon (3 J/ $\mathrm{m}^{2}[8]$ ). For silicon, however, $G_{c}$ is nearly equal to $W$ $\left(2.4 \mathrm{~J} / \mathrm{m}^{2}\right.$ [8]), whereas here less than $10 \%$ of $G_{c}$ is reversible. Our estimated $G_{c}$ is consistent with the expectation [38] that the reversible component of $G_{C}$ is simply the surface energy, $W=2 \gamma=0.14 \mathrm{~J} / \mathrm{m}^{2}$, of the new airwater interfaces created during cracking. A constant $G_{C}$ would imply that $K_{C} / E \sim a^{1 / 6}$, a scaling that may be measurable with a larger range of particle sizes than used here.

We have shown how a well-defined fracture toughness, or fracture energy, can be measured through the deformation fields of drying colloidal films, as long as plasticity is accounted for and care is taken to separate the reversible and irreversible strains. The relative importance of this plasticity should scale with the ratio of surface energies of the dispersant to the colloidal particles. Plastic yielding will therefore be a common feature of desiccation cracks in colloidal materials, which could be exploited to increase their fracture toughness.

We thank R. Oliver for assistance with the AFM, and M. O'Sullivan for assistance with colloidal synthesis. Funding from the Engineering and Physical Sciences Research Council [EP/E05949X/1] is acknowledged.

*lucas.goehring@ds.mpg.de

†wjc1000@cam.ac.uk

tafr10@cam.ac.uk

[1] R. C. Chiu, T. J. Garino, and M. J. Cima, J. Am. Ceram. Soc. 76, 2257 (1993).

[2] C. Allain and L. Limat, Phys. Rev. Lett. 74, 2981 (1995).

[3] L. Goehring, W. J. Clegg, and A. F. Routh, Soft Matter 7, 7984 (2011).

[4] G. Gauthier, V. Lazarus, and L. Pauchard, Europhys. Lett. 89, 26002 (2010).

[5] Z. Néda, K.-t. Leung, L. Józsa, and M. Ravasz, Phys. Rev. Lett. 88, 095502 (2002).

[6] V. Lazarus and L. Pauchard, Soft Matter 7, 2552 (2011).

[7] Y. Xu, W. C. Engl, E. R. Jerison, K. J. Wallenstein, C. Hyland, L. A. Wilen, and E. R. Dufresne, Proc. Natl. Acad. Sci. U.S.A. 107, 14964 (2010).

[8] B.R. Lawn, Fracture of Brittle Solids (Cambridge University Press, Cambridge, England, 1993), 2nd ed., p. 378.
[9] J. W. Hutchinson and Z. Suo, Adv. Appl. Mech. 29, 63 (1991).

[10] J. Zarzycki, J. Non-Cryst. Solids 100, 359 (1988).

[11] A. Sarkar and M.S. Tirumkudulu, Soft Matter 7, 8816 (2011).

[12] D. M. Holmes, F. Tegeler, and W. J. Clegg, J. Eur. Ceram. Soc. 28, 1381 (2008).

[13] S. Kitsunezaki, J. Phys. Soc. Jpn. 78, 064801 (2009).

[14] C. Gurney and J. Hunt, Proc. R. Soc. A 299, 508 (1967).

[15] S. S. L. Peppin, J. A. Elliott, and M. G. Worster, Phys. Fluids 17, 053301 (2005).

[16] A. F. Routh and W. B. Russel, AIChE J. 44, 2088 (1998).

[17] L. Goehring, W. J. Clegg, and A. F. Routh, Langmuir 26, 9269 (2010)

[18] R. C. Chiu and M. J. Cima, J. Am. Ceram. Soc. 76, 2769 (1993).

[19] A. M. König, E. Bourgeat-Lami, V. Mellon, K. von der Ehe, A.F. Routh, and D. Johannsmann, Langmuir 26, 3815 (2010).

[20] M. A. Biot, J. Appl. Phys. 12, 155 (1941).

[21] G. W. Scherer, J. Non-Cryst. Solids 109, 171 (1989).

[22] J. L. Beuth Jr., Int. J. Solids Struct. 29, 1657 (1992).

[23] Z. C. Xia and J. W. Hutchinson, J. Mech. Phys. Solids 48, 1107 (2000)

[24] H. N. Yow, M. Goikoetxea, L. Goehring, and A. F. Routh, J. Colloid Interface Sci. 352, 542 (2010).

[25] J. Leng, Phys. Rev. E 82, 021405 (2010).

[26] J. Li, B. Cabane, M. Sztucki, J. Gummel, and L. Goehring, Langmuir 28, 200 (2012).

[27] E. R. Dufresne, D. J. Stark, N. A. Greenblatt, J. X. Cheng, J.W. Hutchinson, L. Mahadevan, and D. A. Weitz, Langmuir 22, 7144 (2006).

[28] P. C. Kapur, P. J. Scales, D. V. Boger, and T. W. Healy, AIChE J. 43, 1171 (1997).

[29] P. J. Scales, S. B. Johnson, T. W. Healy, and P. C. Kapur, AIChE J. 44, 538 (1998).

[30] G. V. Franks and F. F. Lange, AIChE J. 45, 1830 (1999).

[31] G. V. Franks and F. F. Lange, J. Eur. Ceram. Soc. 21, 893 (2001).

[32] A. Nakahara and Y. Matsuo, Phys. Rev. E 74, 045102 (2006).

[33] W. B. Russel, D. A. Saville, and W. R. Schowalter, Colloidal Dispersions (Cambridge University Press, Cambridge, England, 1989), p. 525.

[34] D. S. Rimai, D. J. Quesnel, and A. A. Busnaina, Colloids Surf. A 165, 3 (2000).

[35] K. Kendall, N. M. Alford, and J. D. Birchall, Proc. R. Soc. A 412, 269 (1987)

[36] C. Thornton, J. Phys. D 26, 1587 (1993).

[37] J. F. Rudd, in Polymer Handbook, edited by J. Brandrup and E.H. Immergud (John Wiley \& Sons, New York, 1975), 2nd ed.

[38] M. S. Tirumkudulu and W. B. Russel, Langmuir 21, 4938 (2005). 